\title{
ADITIVOS POLIMÉRICOS COMO FATOR DE PROTEÇÃO E REABILITAÇÃO DE FLUIDOS HIDROARGILOSOS
}

\author{
L. V. Amorim ${ }^{1}$ \\ E. Pereira ${ }^{2}$ \\ C. M. Gomes ${ }^{3}$ \\ J. D. Viana ${ }^{3}$ \\ K. V. Farias ${ }^{3}$ \\ M. I. R. Barbosa ${ }^{3}$ \\ K. B. França ${ }^{4}$ \\ H. L. Lira \\ H. C. Ferreira ${ }^{5}$ \\ RESUMO
}

\begin{abstract}
Este trabalho tem como objetivo apresentar um estudo realizado em escala de laboratório visando avaliar a ação de alguns aditivos poliméricos na proteção e reabilitação de fluidos hidroargilosos contaminados com cloretos de cálcio e magnésio. Foram preparados fluidos com concentração de 4,86 \% em massa de argila, segundo as normas da Petrobras, e estudadas quatro amostras de argilas bentoníticas sódicas, sendo três delas obtidas em laboratório e uma industrializada em Campina Grande, PB. Foram estudadas amostras de carboximetilcelulose (CMC) de baixa e alta viscosidade e uma amostra de celulose polianiônica (PAC). Os fluidos foram tratados com o aditivo polimérico e após $24 \mathrm{~h}$, foram aditivados com cloretos de cálcio e magnésio (aditivo degradante). Foi também realizada a aditivação em ordem inversa; inicialmente os fluidos foram tratados com o aditivo degradante, e após $24 \mathrm{~h}$, com o aditivo polimérico. A seguir, foram determinadas as viscosidades aparente (VA) e plástica (VP) em viscosímetro Fann 35A e o volume de filtrado (VF) em filtro prensa Fann. Os resultados mostraram a viabilidade do uso de aditivos poliméricos na proteção e na reabilitação de fluidos hidroargilosos, bem como que há indicações da necessidade de modificações nas misturas/proporções das argilas utilizadas pelas empresas beneficiadoras de minérios.
\end{abstract}

Palavras-chave: Fluidos hidroargilosos, proteção, reabilitação, aditivos poliméricos.

\begin{abstract}
The aim of this paper is present a study developed in laboratorial scale to evaluate the action of polymeric additives to protect and recover water and clay based drilling fluids contaminated by calcium and magnesium clorets. Drilling fluids with $4.86 \% \mathrm{w} / \mathrm{w}$ was prepared in accordance to Petrobras standards. Four samples of sodium bentonite clays were tested, three among then was obtained in laboratory and one supplied form industry of state of Paraíba. The drilling fluids were treated with polymeric additive (low and high viscosity of CMC and PAC) and after $24 \mathrm{~h}$, calcium and magnesium clorets were added. Also, effect to inverse sequence was evaluated, that is, first calcium and magnesium clorets were added to the drilling fluids and $24 \mathrm{~h}$ later they were treated with $\mathrm{CMC}$ and PAC. After that, apparent (AV) and plastic (PV) viscosity were measured in viscosimeter Fann 35A and the water loss $(\mathrm{WL})$ in presses filter Fann model. The results shown that polymeric additives can be successfully applied to protect and recover water and clay based drilling fluids. Finally, it is necessary change the blends/proportions of clays actually used by industries.
\end{abstract}

Key-words: Water and clay based drilling fluids, protection, recover, polymeric additives.

\footnotetext{
${ }^{1}$ Doutorado em Eng. de Processos/CCT/UFCG - Bolsista da ANP/PRH-25 - luciana@labdes.ufcg.edu.br

2 Diretor Técnico da Empresa System Mud Indústria e Comércio Ltda. - eugenio@systemmud.com.br

${ }^{3}$ Graduação em Eng. de Materiais/CCT/UFCG

${ }^{4}$ Professor do DEQ/CCT/UFCG - kepler@labdes.ufcg.edu.br

${ }^{5}$ Professor do DEMa/CCT/UFCG - helio@dema.ufcg.edu.br, heber@dema.ufcg.edu.br
} 


\section{Introdução}

Os maiores depósitos de argilas bentoníticas do Brasil estão localizados no Estado da Paraíba, Município de Boa Vista. Essas argilas foram descobertas no início dos anos 60 e ficaram conhecidas pela suas cores características e sua potencialidade de transformar-se em sódicas quando tratadas com carbonato de sódio $\left(\mathrm{Na}_{2} \mathrm{CO}_{3}\right)$. Com esta descoberta, teve início o processo de industrialização das argilas, sendo produzidas matérias primas para diversos fins.

Nos primeiros anos de exploração, era possível encontrar argilas de tonalidades rósea, verde, vermelha, creme, amarela, cinza e chocolate (Souza Santos, 1968). Atualmente, após 40 anos de exploração, muitas das argilas encontram-se esgotadas e outras começando a rarear, a exemplo da argila Chocolate, considerada como de boa qualidade. Ainda em grande quantidade, podem ser encontradas as argilas Bofe (argila de cor creme) e Verde-lodo. Estas argilas, embora possuam composição mineralógica semelhante, sendo compostas por argilominerais esmectíticos e impurezas de quartzo, apresentam comportamento reológico bastante variado. A argila Verdelodo apresenta ainda ilita e caulinita (Amorim e Pereira, 2003).

Segundo os últimos dados do Departamento Nacional de Produção Mineral - DNPM (Trindade, 2001), as reservas de Boa Vista totalizam cerca de 16 milhões de toneladas de argila e a quantidade de bentonita bruta e beneficiada (bentonita sódica) produzida na Paraíba representa $96 \%$ da produção nacional. Os $4 \%$ restantes são produzidas no Estado de São Paulo.

Os produtos industrializados são misturas de diversas espécies de argila, em proporções variadas, que condicionam suas aplicações. Hoje, são produzidas na Paraíba bentonitas para areias de fundição, pelotização de minério de ferro, rejeito de dejetos animais, clarificantes de óleos, na indústria química e farmacêutica e como agente viscosificante e tixotrópico em fluidos de perfuração de poços de petróleo e tubulares.

Nas operações de perfuração de poços são freqüentes os problemas provocados pela contaminação do fluido de perfuração. 10
Essa contaminação pode ser causada pelo uso de águas impróprias utilizadas no preparo do fluido ou proveniente das formações geológicas que estão sendo perfuradas. No primeiro caso, muitas das águas industriais, de rios, lagos ou de poços tubulares perfurados especialmente para servirem de fonte de água para o preparo do fluido, possuem sais que inibem 0 inchamento da argila, prejudicando suas propriedades, ou seja, viscosidade, gel e controle de filtrado e reboco. No segundo caso, a dissolução de íons provenientes das formações geológicas também prejudicam as propriedades do fluido através da floculação da dispersão hidroargilosa.

Embora a contaminação dos fluidos de perfuração conduza a sérios problemas, poucos têm sido os estudos sistemáticos que indiquem soluções, como, por exemplo, o tratamento dos fluidos com aditivos específicos antes do início da sua contaminação.

Este trabalho tem como objetivo apresentar um estudo realizado em escala de laboratório visando avaliar a ação de alguns aditivos poliméricos na proteção e reabilitação de fluidos hidroargilosos contaminados com cloretos de cálcio e magnésio.

\section{Materiais}

\subsection{Argilas Bentoníticas}

Foram estudadas quatro amostras de argilas bentoníticas sódicas, sendo três delas obtidas em laboratório, denominadas de Bofe, Chocolate e Verde-lodo e uma industrializada em Campina Grande, PB, pela Empresa Bentonit União Nordeste BUN, comercialmente conhecida por Brasgel PA. As amostras de argilas naturais e as amostras que compõem a argila industrializada são provenientes das jazidas do Município de Boa Vista, PB.

\subsection{Aditivo de Proteção e Reabilitação}

Foram estudadas duas amostras de carboximetilcelulose (CMC) com diferentes graus de viscosidade, CMC BV, CMC de baixa viscosidade e CMC AV, CMC de alta viscosidade e uma amostra de PAC (celulose polianiônica). Na Tabela 1 , estão apresentados os dados técnicos dos aditivos. O CMC BV foi estudado nas 
concentrações de $0,3 \mathrm{~g} / 24,3 \mathrm{~g}$ de argila seca, 0,4 g/ 24,3 g de argila seca e 0,5 $\mathrm{g} / 24,3 \mathrm{~g}$ de argila seca, enquanto que 0 CMC AV e o PAC foram estudados nas concentrações de 0,2 g/24,3 g de argila seca, 0,3 g/24,3 $\mathrm{g}$ de argila seca e 0,4 $\mathrm{g} / 24,3 \mathrm{~g}$ de argila seca. Os aditivos foram fornecidos pela Empresa System Mud Indústria e Comércio Ltda.

Tabela 1 - Dados técnicos dos aditivos poliméricos.

\begin{tabular}{cccc}
\hline Dados & \multicolumn{3}{c}{ Amostras } \\
\cline { 2 - 4 } Técnicos & CMC BV & CMC AV & PAC \\
\hline Função & $\begin{array}{c}\text { Viscosificante e } \\
\text { Redutor de Filtrado }\end{array}$ & $\begin{array}{c}\text { Viscosificante e } \\
\text { Redutor de Filtrado }\end{array}$ & $\begin{array}{c}\text { Viscosificante e } \\
\text { Redutor de Filtrado }\end{array}$ \\
\hline Aplicação & Água Doce & Água Doce & Água Salgada \\
\hline Composição & CMC de Sódio & CMC de Sódio & Celulose Polianiônica \\
\hline Tamanho de Cadeia & Longa & Longa & Longa \\
\hline Grau de Substituição & 0,75 & 0,85 & 0,95 \\
\hline Pureza (\%) & $>95,00$ & $>95,00$ & $>98,00$ \\
\hline Peso Molecular & $>250.000$ & $>700.000$ & $>700.000$ \\
\hline
\end{tabular}

\subsection{Aditivo de Degradação}

Foram estudados o cloreto de cálcio PA $\left(\mathrm{CaCl}_{2}\right)$ e o cloreto de magnésio PA $\left(\mathrm{MgCl}_{2}\right)$ da marca VETEC. Esses aditivos foram adicionados simultaneamente na concentração de 10 meq de $\mathrm{CaCl}_{2} / 100 \mathrm{~g}$ de argila seca +10 meq de $\mathrm{MgCl}_{2} / 100 \mathrm{~g}$ de argila seca.

\section{Metodologia}

\subsection{Transformação da Argila Bentonítica Natural em Sódica}

As argilas naturais Bofe, Chocolate e Verde-lodo foram aditivadas com solução concentrada de $\mathrm{Na}_{2} \mathrm{CO}_{3}(0,2 \mathrm{~g} / \mathrm{mL})$ nas proporções de 150 meq de $\mathrm{Na}_{2} \mathrm{CO}_{3} / 100 \mathrm{~g}$ de argila seca, 75 meq de $\mathrm{Na}_{2} \mathrm{CO}_{3} / 100 \mathrm{~g}$ de argila seca e 100 meq de $\mathrm{Na}_{2} \mathrm{CO}_{3} / 100 \mathrm{~g}$ de argila seca, respectivamente. Após $05 \mathrm{~d}$ de cura em câmara úmida com $100 \%$ de umidade relativa, foram preparados os fluidos de perfuração como descrito no item 3.2.

\subsection{Preparação dos Fluidos de Perfuração}

Os fluidos de perfuração foram preparados com concentração de 4,86 \% em massa de argila (24,3 g de argila em $500 \mathrm{~mL}$ de água deionizada), de acordo com a norma N-2605 (Petrobras, 1998).

\subsection{Tratamento de Proteção}

Após preparação, os fluidos de perfuração foram tratados com os aditivos poliméricos e agitados durante $5 \mathrm{~min}$. Após $24 \mathrm{~h}$, foram tratados com $\mathrm{o}$ aditivo de degradação $\left(\mathrm{CaCl}_{2}+\mathrm{MgCl}_{2}\right)$ e novamente agitados durante $5 \mathrm{~min}$, permanecendo em repouso por mais $24 \mathrm{~h}$. Em seguida, foi realizado o estudo reológico, como descrito no item 3.5.

\subsection{Tratamento de Reabilitação}

Para o tratamento de reabilitação, o aditivo de degradação $\left(\mathrm{CaCl}_{2}+\mathrm{MgCl}_{2}\right)$ foi adicionado à água e, em seguida, foi adicionada a argila sob agitação, sendo o fluido preparado de acordo com o item 3.2. Após $24 \mathrm{~h}$, os fluidos foram tratados com os aditivos poliméricos e agitados por $5 \mathrm{~min}$, permanecendo em repouso por mais $24 \mathrm{~h}$. Em seguida, foi realizado o estudo reológico, como descrito no item 3.5.

\subsection{Estudo Reológico}

Foram determinadas as viscosidades aparente (VA) e plástica (VP), em viscosímetro Fann 35A e o volume de filtrado em filtro Prensa Fann, segundo a norma N-2605 (Petrobras, 1998).

\section{Resultados e Discussão}

Através dos resultados obtidos quando da adição dos aditivos poliméricos aos fluidos, observou-se comportamento diferenciado para cada uma das argilas estudadas. Para a argila Bofe na forma sódica, que possui reologia inicial fora das especificações da PETROBRAS (1998a), observou-se, após aditivação com o CMC BV, o CMC AV e o PAC, melhoria no seu rendimento reológico, atingindo valores de VA, VP e VF de acordo com os limites 
especificados para uso na perfuração de poços de petróleo. Os resultados obtidos com o CMC BV e com o PAC estão apresentados nas Tabelas 2 e 3 e nas Figuras 1(a) e 1(b), respectivamente.

Os fluidos preparados com a argila Chocolate na forma sódica, apresentam elevada VA $(18,5 \mathrm{cP})$, baixa VP $(3,8 \mathrm{cP})$ e VF da ordem de $16,0 \mathrm{~mL}$. Após a adição dos aditivos poliméricos, observou-se um acréscimo acentuado em $V A$, pequenas variações em VP e diminuição em VF. Esse comportamento indesejável é característico de um sistema floculado, provavelmente, devido à formação de pontes, ou seja, as cadeias poliméricas do aditivo são adsorvidas às superfícies das partículas de argila, diminuindo as distâncias entre elas e promovendo o fenômeno de floculação. A formação de pontes também pode ocorrer através de interações laterais entre os grupos hidrofóbicos do polímero. Essas interações aproximam as partículas de argila, resultando na sua floculação.

Os fluidos preparados com a argila Verde-lodo na forma sódica, por sua vez, desenvolvem sistemas denominados de floculado-gel, traduzido pelos valores muito elevados de VA e VF, e muito baixos de VP, próximos de 1,0 cP. Neste estado, as partículas de argila permanecem unidas através de interações elétricas e de massa, com associações do tipo face-a-aresta e aresta-a-aresta, gerando uma estrutura do tipo castelo-de-cartas, no qual, todo o volume do líquido é ocupado pelas partículas de argila (Souza Santos, 1989). Após adição dos aditivos poliméricos, observou-se uma transição do estado floculado-gel para o estado floculado (estado no qual as interações predominantes entre as partículas de argila são do tipo face-aface), contudo, os resultados obtidos são pouco satisfatórios, não atendendo aos limites especificados pela Petrobras (1998a). Para os fluidos preparados com a argila industrializada, observou-se que a adição dos aditivos poliméricos promoveu aumento nos valores de VA e VP, e diminuição nos valores de VF. As Tabelas 4 e 5 e as Figuras 2(a) e 2(b), apresentam os resultados obtidos quando da aditivação com O CMC BV e com o PAC, respectivamente.

O aumento de viscosidade e a redução no volume de filtrado, obtidos quando da simples aditivação com os polímeros era esperado, uma vez que os aditivos estudados têm como função melhorar a reologia e reduzir as perdas por filtração. Em particular, observou-se que o volume de filtrado é sensível ao tipo de polímero utilizado, sendo os menores valores obtidos quando da aditivação com o PAC. Esse comportamento é contrário ao observado em estudos realizados com fluidos hidroargilosos, aditivados com diferentes amostras de CMC e de PAC (Hughes et al., 1993).

Tabela 2 - Propriedades reológicas dos fluidos preparados com a argila Bofe na forma sódica após aditivação e após os tratamentos de degradação, proteção e reabilitação com CMC BV.

\begin{tabular}{|c|c|c|c|c|c|}
\hline Tratamentos & Concentrações & VA (cP) & $\mathrm{VP}(\mathrm{cP})$ & $\begin{array}{l}\text { VF } \\
(\mathrm{mL})\end{array}$ & $\mathrm{pH}$ \\
\hline $\mathrm{Na}_{2} \mathrm{CO}_{3}$ & $\begin{array}{c}150 \mathrm{meq} / 100 \mathrm{~g} \text { de argila } \\
\text { seca }\end{array}$ & 12,5 & 4,8 & 17,7 & 10,0 \\
\hline $\begin{array}{c}\text { Degradação }\left(\mathrm{CaCl}_{2}+\right. \\
\left.\mathrm{MgCl}_{2}\right)\end{array}$ & 10 meq + 10 meq & 10,8 & 4,5 & 17,8 & 10,0 \\
\hline \multirow{3}{*}{ CMC BV } & $0,3 \mathrm{~g}$ & 13,3 & 7,0 & 17,3 & 10,0 \\
\hline & $0,4 \mathrm{~g}$ & 15,5 & 8,0 & 17,2 & 10,1 \\
\hline & $0,5 \mathrm{~g}$ & 18,5 & 7,0 & 16,3 & 10,0 \\
\hline Proteção & $0,3 \mathrm{~g}$ & 15,3 & 7,0 & 17,5 & 9,8 \\
\hline (CMC BV + aditivo de & $0,4 \mathrm{~g}$ & 17,3 & 7,0 & 16,0 & 9,8 \\
\hline $\begin{array}{c}\text { degradação }(10+10 \\
\text { meq)) }\end{array}$ & $0,5 \mathrm{~g}$ & 20,5 & 7,0 & 15,3 & 9,8 \\
\hline Reabilitação & $0,3 \mathrm{~g}$ & 17,5 & 6,5 & 18,0 & 9,7 \\
\hline (aditivo de degradação & $0,4 \mathrm{~g}$ & 18,3 & 8,5 & 16,0 & 9,8 \\
\hline$(10+10$ meq $)+$ CMC BV $)$ & $0,5 \mathrm{~g}$ & 19,0 & 8,0 & 16,3 & 9,8 \\
\hline \multicolumn{2}{|c|}{ Especificações N-2604 (Petrobras, 1998) } & $\geq 15,0$ & $\geq 4,0$ & $\leq 18,0$ & $\leq 10,0$ \\
\hline
\end{tabular}


Rev. Águas Subterrâneas nº 18/ Janeiro 2004 
Tabela 3 - Propriedades reológicas dos fluidos preparados com a argila Bofe na forma sódica após aditivação e após os tratamentos de degradação, proteção e reabilitação com PAC.

\begin{tabular}{|c|c|c|c|c|c|}
\hline Tratamentos & Concentrações & $\mathrm{VA}(\mathrm{cP})$ & $\mathrm{VP}(\mathrm{cP})$ & $\begin{array}{l}\mathrm{VF} \\
(\mathrm{mL})\end{array}$ & $\mathrm{pH}$ \\
\hline $\mathrm{Na}_{2} \mathrm{CO}_{3}$ & $\begin{array}{c}150 \text { meq/100 g de argila } \\
\text { seca }\end{array}$ & 12,5 & 4,8 & 17,7 & 10,0 \\
\hline $\begin{array}{c}\text { Degradação }\left(\mathrm{CaCl}_{2}+\right. \\
\left.\mathrm{MgCl}_{2}\right)\end{array}$ & 10 meq + 10 meq & 10,8 & 4,5 & 17,8 & 10,0 \\
\hline \multirow{3}{*}{ PAC } & $0,2 \mathrm{~g}$ & 12,8 & 5,0 & 14,0 & 10,2 \\
\hline & $0,3 \mathrm{~g}$ & 14,0 & 5,5 & 12,3 & 10,2 \\
\hline & $0,4 \mathrm{~g}$ & 15,0 & 6,5 & 11,5 & 10,2 \\
\hline Proteção & $0,2 \mathrm{~g}$ & 17,0 & 5,0 & 13,5 & 9,9 \\
\hline (PAC + aditivo de & $0,3 \mathrm{~g}$ & 16,3 & 6,0 & 13,3 & 9,8 \\
\hline $\begin{array}{c}\text { degradação } \\
(10+10 \text { meq) })\end{array}$ & $0,4 \mathrm{~g}$ & 17,5 & 6,0 & 12,5 & 9,8 \\
\hline Reabilitação & $0,2 \mathrm{~g}$ & 15,0 & 5,5 & 15,8 & 9,8 \\
\hline (aditivo de degradação & $0,3 \mathrm{~g}$ & 17,8 & 6,0 & 13,0 & 9,8 \\
\hline$(10+10 \mathrm{meq})+\mathrm{PAC})$ & $0,4 \mathrm{~g}$ & 17,5 & 6,0 & 12,5 & 9,7 \\
\hline \multicolumn{2}{|c|}{ Especificações N-2604 (Petrobras, 1998) } & $\geq 15,0$ & $\geq 4,0$ & $\leq 18,0$ & $\leq 10,0$ \\
\hline
\end{tabular}

A redução do volume de filtrado pela introdução de aditivos poliméricos em fluidos de perfuração à base de água é explicada através dos mecanismos de estabilização eletrostática e de estabilização eletroestérica. A estabilização eletrostática ocorre como conseqüência da repulsão entre as cadeias do polímero adsorvidas às superfícies das partículas de argila, enquanto que, a estabilização eletroestérica, ocorre como conseqüência do aumento da distância mínima de separação entre as partículas de argila que estão envolvidas pelas cadeias poliméricas e pelas interações de caráter elétrico repulsivo entre as cadeias do polímero (Oliveira et al., 2000). Esses mecanismos evitam o fenômeno de floculação das partículas de argila, obtendose sistemas com as partículas de argila dispersas no meio líquido.

Quando do tratamento dos fluidos apenas com o aditivo de degradação $\left(\mathrm{CaCl}_{2}\right.$ $+\mathrm{MgCl}_{2}$ ), observou-se para todas as amostras de argilas estudadas, a perda de suas propriedades reológicas. Esse comportamento deve-se à sensibilidade do sistema argila bentonítica-água aos íons cálcio e magnésio, que quando presentes, diminuem a repulsão eletrostática entre as partículas de argila, promovendo a sua agregação (floculação), com redução das suas propriedades reológicas.

Através dos resultados obtidos quando do tratamento de proteção dos fluidos com os aditivos poliméricos frente ao agente de degradação $\left(\mathrm{CaCl}_{2}+\mathrm{MgCl}_{2}\right)$, observou-se, da mesma forma quando da simples aditivação com os polímeros, comportamento diferenciado para cada uma das argilas estudadas. Os melhores resultados foram obtidos para os fluidos preparados com a argila Bofe na forma sódica aditivados com o CMC BV (Tabela 2 e Figura 1(a)) e com o PAC (Tabela 3 e Figura 1(b)). Através dos resultados, observou-se que tanto o CMC BV quanto o PAC, protegem os fluidos da ação floculante do cálcio e do magnésio, apresentando propriedades reológicas que satisfazem as especificações da Petrobras (1998a), para uso na perfuração de poços de petróleo. Os resultados obtidos com O CMC AV evidenciaram que sua ação é limitada frente ao agente de degradação, pois embora as viscosidades apresentem valores adequados, os valores de VF mantiveram-se superiores a $18,0 \mathrm{~mL}$, máximo especificado pela Petrobras (1998a).

Para os fluidos preparados com as argilas Chocolate e Verde-lodo nas formas sódicas, os resultados mostraram que os aditivos poliméricos não protegem os fluidos da ação floculante do cálcio e do magnésio. Após o tratamento de proteção, os fluidos apresentaram comportamento reológico característico de um sistema floculado. O mesmo comportamento foi observado para os fluidos preparados com a argila industrializada (Tabelas 4 e 5 e Figuras 2(a) e 2(b)). 
Figura 1 - Propriedades reológicas dos fluidos preparados com a argila Bofe na forma sódica após aditivação e após os tratamentos de degradação, proteção e reabilitação com o (a) CMC BV e com o (b) PAC.

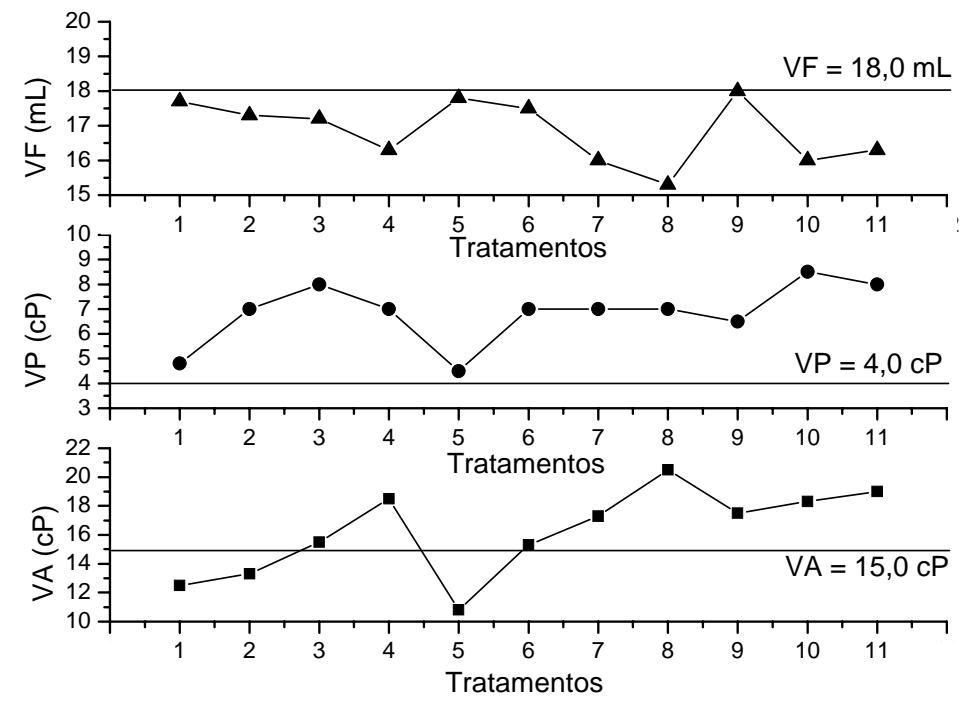

(a)

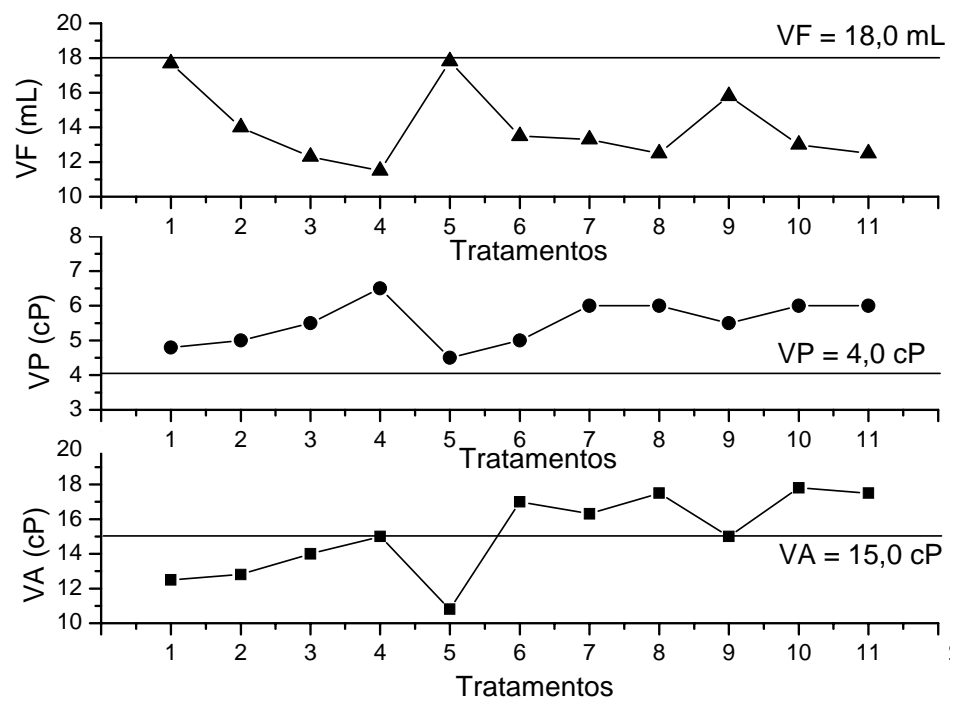

(b)
Legenda

Tratamentos

1 - Sem aditivação

2 - 0,3 g de CMC BV

$3-0,4 \mathrm{~g}$ de CMC BV

4 - $0,5 \mathrm{~g}$ de CMC BV

5 - Degradação (AD)

(10 meq de $\mathrm{CaCl}_{2}+10$ meq de $\mathrm{MgCl}_{2}$ )

$6-0,3 \mathrm{~g}$ de $C M C^{2} B V+A D$

$7-0,4 \mathrm{~g}$ de $C M C B V+A D$

$8-0,5 \mathrm{~g}$ de $C M C B V+A D$

$9-\mathrm{AD}+0,3 \mathrm{~g}$ de CMC BV

$10-A D+0,4 \mathrm{~g}$ de $\mathrm{CMC} B \mathrm{BV}$

$11-A D+0,5 \mathrm{~g}$ de $C M C B V$
Legenda

Tratamentos

1 - Sem aditivação

2 - $0,2 \mathrm{~g}$ de PAC

$3-0,3 \mathrm{~g}$ de PAC

4 - $0,4 \mathrm{~g}$ de PAC

5 - Degradação (AD)

10 meq de $\mathrm{CaCl}_{2}+10$ meq de $\mathrm{MgCl}_{2}$

$6-0,2 \mathrm{~g}$ de $P A C+A D$

7 - 0,3 g de PAC + AD

$8-0,4 \mathrm{~g}$ de $P A C+A D$

$9-A D+0,2 \mathrm{~g}$ de $P A C$

$10-A D+0,3 \mathrm{~g}$ de $P A C$

$11-A D+0,4 \mathrm{~g}$ de $P A C$
Através dos resultados obtidos quando do tratamento de reabilitação dos fluidos degradados com $\mathrm{CaCl}_{2}+\mathrm{MgCl}_{2}$, observouse o mesmo comportamento obtido no tratamento de proteção, ou seja, os melhores resultados foram obtidos para os fluidos preparados com a argila Bofe na forma sódica, aditivados com o CMC BV (Tabela 2 e Figura 1(a)) e com o PAC (Tabela 3 e Figura 1(b)). Através dessas Figuras, observou-se que tanto o CMC BV quanto o PAC recuperam as propriedades reológicas dos fluidos contaminados com cálcio e magnésio, com valores de VA, VP e VF de acordo com os limites especificados pela Petrobras (1998a). Os resultados obtidos com o CMC AV evidenciaram que sua ação é também limitada na reabilitação de fluidos contaminados.

Para os fluidos preparados com as argilas Chocolate e Verde-lodo nas formas sódicas, os resultados mostraram que os 
aditivos poliméricos não recuperam as propriedades reológicas dos fluidos contaminados. Após o tratamento de reabilitação, os fluidos apresentaram reologias características de um sistema floculado. O mesmo comportamento foi observado para os fluidos preparados com a argila industrializada (Tabelas 4 e 5 e Figuras 2(a) e 2(b)).

Através dos resultados apresentados acima, observou-se a eficiência do CMC BV e do PAC na proteção e na reabilitação de fluidos hidroargilosos contaminados com cálcio e magnésio. No tratamento de proteção, as partículas de argilas são estabilizadas pela formação de uma película polimérica que as protege da ação floculante do cálcio e do magnésio. No tratamento de reabilitação, a recuperação das propriedades reológicas dos fluidos ocorre devido a adsorção do polímero nas partículas de argila, que aumenta as forças de repulsão, diminuindo, assim, o grau de floculação. Também nestes casos, estão envolvidos os mecanismos de repulsão eletrostática, devido às cargas negativas dos polímeros, e de repulsão eletroestérica entre as camadas de polímero adsorvidas. Segundo Meadows et al. (1989), as cadeias poliméricas adsorvidas à superfície das argilas na presença de eletrólitos, apresentam uma conformação do tipo espiral, diminuindo assim a interação eletrostática, contudo, o efeito de floculação é vencido pela maior quantidade de polieletrólito adsorvido, devido o enfraquecimento da repulsão intermolecular.

Tabela 4 - Propriedades reológicas dos fluidos preparados com a argila industrializada após aditivação e após os tratamentos de degradação, proteção e reabilitação com CMC BV.

\begin{tabular}{|c|c|c|c|c|c|}
\hline Tratamentos & Concentrações & VA (cP) & $\mathrm{VP}(\mathrm{cP})$ & $\begin{array}{l}\mathrm{VF} \\
(\mathrm{mL})\end{array}$ & $\mathrm{pH}$ \\
\hline Sem aditivação & $\begin{array}{c}----- \\
-\cdots\end{array}$ & 16,1 & 4,0 & 16,3 & 9,9 \\
\hline $\begin{array}{c}\text { Degradação }\left(\mathrm{CaCl}_{2}+\right. \\
\left.\mathrm{MgCl}_{2}\right)\end{array}$ & 10 meq + 10 meq & 16,8 & 3,5 & 19,8 & 9,3 \\
\hline \multirow{3}{*}{ CMC BV } & $0,3 \mathrm{~g}$ & 20,8 & 7,0 & 14,8 & 9,3 \\
\hline & $0,4 \mathrm{~g}$ & 25,5 & 6,5 & 15,0 & 9,1 \\
\hline & $0,5 \mathrm{~g}$ & 27,8 & 6,5 & 13,5 & 9,0 \\
\hline Proteção & $0,3 \mathrm{~g}$ & 36,3 & 3,5 & 17,5 & 8,8 \\
\hline (CMC BV +aditivo de & $0,4 \mathrm{~g}$ & 43,5 & 4,5 & 17,0 & 8,2 \\
\hline $\begin{array}{c}\text { degradação }(10+10 \\
\text { meq)) }\end{array}$ & $0,5 \mathrm{~g}$ & 46,8 & 3,0 & 15,5 & 8,4 \\
\hline Reabilitação & $0,3 \mathrm{~g}$ & 29,5 & 5,0 & 18,0 & 8,6 \\
\hline (aditivo de degradação & $0,4 \mathrm{~g}$ & 32,5 & 3,0 & 16,0 & 8,5 \\
\hline$(10+10 \mathrm{meq})+\mathrm{CMC} \mathrm{BV})$ & $0,5 \mathrm{~g}$ & 35,8 & 3,5 & 15,5 & 8,5 \\
\hline \multicolumn{2}{|c|}{ Especificações N-2604 (Petrobras, 1998) } & $\geq 15,0$ & $\geq 4,0$ & $\leq 18,0$ & $\leq 10,0$ \\
\hline
\end{tabular}

Tabela 5 - Propriedades reológicas dos fluidos preparados com a argila industrializada após aditivação e após os tratamentos de degradação, proteção e reabilitação com PAC.

\begin{tabular}{cccccc}
\hline Tratamentos & Concentrações & VA (cP) & VP (cP) & $\begin{array}{c}\text { VF } \\
(\mathrm{mL})\end{array}$ & $\mathrm{pH}$ \\
\hline Sem aditivação & ----- & 16,1 & 4,0 & 16,3 & 9,9 \\
\hline $\begin{array}{c}\text { Degradação }\left(\mathrm{CaCl}_{2}+\right. \\
\left.\mathrm{MgCl}_{2}\right)\end{array}$ & 10 meq + 10 meq & 16,8 & 3,5 & 19,8 & 9,3 \\
PAC & $0,2 \mathrm{~g}$ & 24,8 & 3,5 & 13,2 & 9,5 \\
& $0,3 \mathrm{~g}$ & 24,0 & 5,0 & 11,5 & 9,5 \\
\hline Proteção & $0,4 \mathrm{~g}$ & 24,8 & 5,5 & 10,8 & 9,5 \\
\hline $\begin{array}{c}\text { (PAC + aditivo de } \\
\text { degradação }\end{array}$ & $0,2 \mathrm{~g}$ & 85,5 & 11,5 & 14,0 & 9,0 \\
$(10+10$ meq) & $0,3 \mathrm{~g}$ & 104,5 & 15,0 & 11,5 & 9,0 \\
Reabilitação & $0,4 \mathrm{~g}$ & 95,5 & 7,5 & 10,2 & 9,0 \\
\hline & $0,2 \mathrm{~g}$ & & & & \\
\hline
\end{tabular}




\begin{tabular}{ccrrrr}
\hline (aditivo de degradação & $0,3 \mathrm{~g}$ & 72,5 & 14,0 & 12,0 & 8,9 \\
$(10+10$ meq) + PAC & $0,4 \mathrm{~g}$ & 53,0 & 5,5 & 11,0 & 8,9 \\
\hline Especificações N-2604 (Petrobras, 1998) & $\geq 15,0$ & $\geq 4,0$ & $\leq 18,0$ & $\leq 10,0$ \\
\hline
\end{tabular}

Em trabalhos anteriores (Amorim et al., 2002 e Amorim et al., 2003), foram apresentados os resultados obtidos com um CMC de baixa viscosidade que age como defloculante e redutor de filtrado. Neste caso, bons resultados foram obtidos para os fluidos preparados com a argila Verde-lodo na forma sódica, sendo possível sua proteção e sua reabilitação quando da utilização de concentrações de CMC da ordem de 1,74 g/24,3 g de argila seca. Para os fluidos preparados com a argila Bofe na forma sódica e com argilas industrializadas da Paraíba, embora o CMC tenha se mostrado eficiente na proteção e na recuperação da reologia dos fluidos, não foram atingidos os limites especificados pela Petrobras (1998a). Para os fluidos preparados com a argila Chocolate na forma sódica, a adição do CMC conduziu o sistema ao estado floculado.

Figura 2 - Propriedades reológicas dos fluidos preparados com a argila industrializada após aditivação e após os tratamentos de degradação, proteção e reabilitação com o (a) CMC BV e com o (b) PAC.
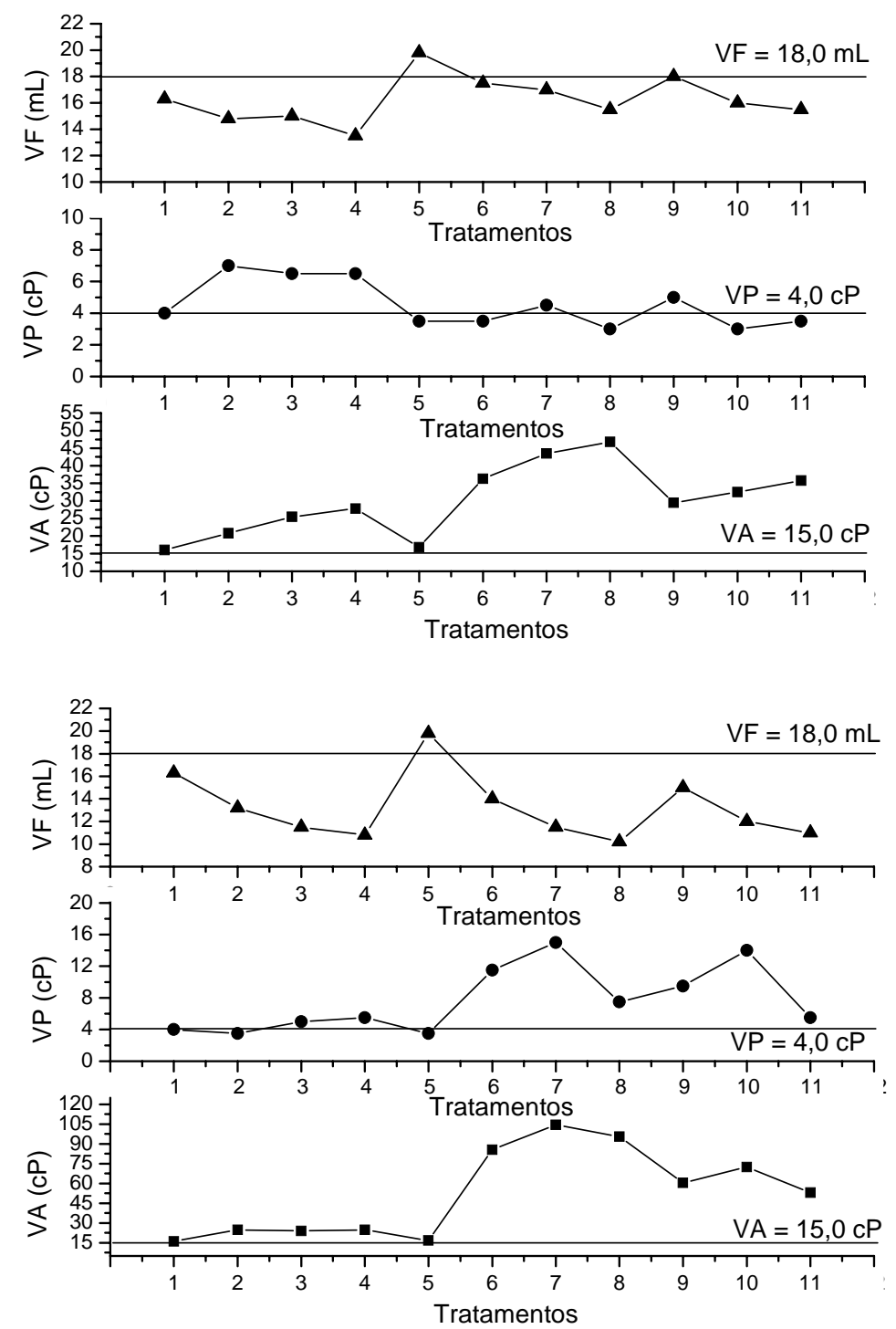

Legenda

Tratamentos

1 - Sem aditivação

2 - 0,3 g de CMC BV

$3-0,4 \mathrm{~g}$ de CMC BV

4 - 0,5 g de CMC BV

5 - Degradação (AD)

10 meq de $\mathrm{CaCl}_{2}+10$ meq de $\mathrm{MgCl}_{2}$

$6-0,3 \mathrm{~g}$ de CMC BV + AD

$7-0,4 \mathrm{~g}$ de $C M C B V+A D$

$8-0,5 \mathrm{~g}$ de $\mathrm{CMC} B \mathrm{BV}+\mathrm{AD}$

$9-\mathrm{AD}+0,3 \mathrm{~g}$ de CMC BV

$10-A D+0,4 \mathrm{~g}$ de $C M C B V$

$11-A D+0,5 \mathrm{~g}$ de $C M C B V$
Legenda

Tratamentos

1 - Sem aditivação

2 - 0,2 g de PAC

$3-0,3 \mathrm{~g}$ de PAC

4 - $0,4 \mathrm{~g}$ de PAC

5 - Degradação (AD)

10 meq de $\mathrm{CaCl}_{2}+10$ meq de $\mathrm{MgCl}_{2}$

$6-0,2 \mathrm{~g}$ de $P A C+A D$

$7-0,3 \mathrm{~g}$ de $P A C+A D$

$8-0,4 \mathrm{~g}$ de $P A C+A D$

$9-A D+0,2 \mathrm{~g}$ de $P A C$

$10-A D+0,3 \mathrm{~g}$ de $P A C$

$11-A D+0,4 \mathrm{~g}$ de $P A C$ 
Um fato de grande importância observado através deste estudo, foi o comportamento diferenciado para cada tipo de argila bentonítica, sendo a Bofe a única a apresentar resultados satisfatórios quando dos tratamentos de proteção e reabilitação. A argila industrializada que apresenta propriedades reológicas e de filtração de acordo com as normas da Petrobras (1998), também não apresentou comportamento satisfatório. Com base nestes resultados, são sugeridas modificações nas misturas das argilas utilizadas pelas empresas beneficiadoras de bentonita, através do uso da argila Bofe em maior concentração, de forma a se obter um produto com propriedades mais adequadas, bem como, a fabricação de bentonitas modificadas com polímeros para uso em perfurações com perfis geológicos que possam ser prováveis fontes de contaminação.

A modificação nas misturas/proporções utilizadas pelas empresas beneficiadoras de bentonita se faz também necessário pela atual situação das jazidas de Boa Vista, PB, que após décadas de exploração, tem suas variedades de argila de melhor qualidade praticamente esgotadas. A falta de planejamento da utilização dessas argilas poderá ocasionar a redução na vida útil das jazidas, com isso, medidas como a busca de composições que utilizem o mínimo das variedades de melhor qualidade e resultem em fluidos com propriedades reológicas adequadas, podem ajudar a minimizar as conseqüências deste problema a médio e a longo prazo.

Uma análise conjunta dos resultados apresentados neste trabalho e em estudos anteriores (Amorim et al., 2002 e Amorim et al., 2003), mostra que não é possível fazer generalizações sobre qual tipo de polímero deverá ser utilizado como aditivo protetor ou de reabilitação de fluidos hidroargilosos contaminados, uma vez que o fator determinante é o tipo de argila bentonítica utilizada no preparo do fluido.

\section{Conclusões}

Com o objetivo de avaliar a ação de alguns aditivos poliméricos na proteção e na reabilitação de fluidos hidroargilosos contaminados com cloretos de cálcio e magnésio, concluiu-se que:

i) a adição do $\mathrm{CMC} \mathrm{BV}$, do $\mathrm{CMC} \mathrm{AV}$ e do PAC melhora o comportamento reológico dos fluidos, promovendo aumento nos 16 valores de VA e VP e diminuição nos valores de VF;

ii) o CMC BV e o PAC agem de forma eficiente na proteção e na reabilitação dos fluidos preparados com a argila Bofe na forma sódica;

iii) em virtude do esgotamento das argilas de melhor qualidade de Boa Vista, PB, e do comportamento reológico apresentado pelos fluidos preparados com a argila industrializada, recomendam-se estudos no sentido de melhor aproveitar as espécies mineralógicas ora existentes, com o objetivo de otimizar a vida útil dos jazimentos; e iv) é viável a obtenção de bentonitas modificadas com polímeros para uso em perfurações de formações geológicas que apresentem possibilidades de contaminação.

\section{Agradecimentos}

Os autores agradecem à Agência Nacional do Petróleo - ANP, ao CNPq/CTPETRO, ao MCT e a FINEP, pelo apoio financeiro para o desenvolvimento desta pesquisa, e à Empresa System Mud Indústria e Comércio Ltda., pelo fornecimento dos aditivos poliméricos.

\section{Referências Bibliográficas}

AMORIM, L.V., GOMES, C.M., FARIAS, K.V., VIANA, J.D., FERREIRA, H.C. Uso do CMC como colóide protetor em fluidos de perfuração à base de água e argila. In: XV Congresso Brasileiro de Ciências e Engenharia de Materiais - CBECIMAT, Natal, Brasil: nov., 2002. No prelo.

AMORIM, L.V., VIANA, J.D., FARIAS, K.V., FRANÇA, K.B., LIRA, H.L., FERREIRA, H.C. Uso do CMC como colóide protetor em fluidos de perfuração à base de água e argilas: Parte II. In: $2^{\circ}$ Congresso Brasileiro de $P$ \& $D$ em Petróleo e Gás, Rio de Janeiro, Brasil: jul., 2003.

AMORIM, L.V., PEREIRA, E. Bentonitas da Paraíba: O Barro Chega a Universidade, Jornal ABAS Informa, São Paulo, maio, v. 138, 2003.

HUGHES, T.L., JONES, T.G.J., O.H., HOUWEN. Checimcal characterization of $\mathrm{CMC}$ and its relationship to drilling-mud rheology and fluid loss, SPE Drilling \& Completion, set., 1993.

MEADOWS, J., WILLIANS, P.A., GARVEY, M.J., HARROP, R., PHILLIPS, G.O., 
Characterization of the adsorptiondesorption behavior of hydrolysed polyacrylamide, J. Colloid Interf. Sci., 132 (2): 319-328, 1989.

OLIVEIRA, I.R., STUDART, A.R., PILEGGI, R.G., PANDOLFELLI, V.C., Dispersão e Empacotamento de partículas, princípios e aplicações em processamento cerâmico, Fazendo Arte Editorial, São Paulo, (2000) 30-31.

PETROBRAS, Argila ativada para fluido de perfuração à base de água na exploração e produção de petróleo, Método, N-2605, 1998.

PETROBRAS, Argila ativada para fluido de perfuração à base de água na exploração e produção de petróleo, Especificação, N2604, 1998a.

SOUZA SANTOS, P., Estudo tecnológico de argilas montmorilonitas do Distrito de Boa Vista, Município de Campina Grande, Paraíba, Tese de concurso à Cátedra de Química Industrial, DEQ, EPUSP, São Paulo, 1968.

SOUZA SANTOS, P., Ciência e Tecnologia de Argilas, vol. I, Editora Edgard Blücher Ltda., São Paulo (1989) 109.

TRINDADE, M.H.A., Bentonitas, Disponível em <www.dnpm.org.br>. Acesso em 30 de janeiro de 2003. 